\title{
Axially Symmetric, Asymptotically Flat Vacuum Metric with a Naked Singularity and Closed Timelike Curves
}

\author{
Debojit Sarma, Faizuddin Ahmed, and Mahadev Patgiri \\ Department of Physics, Cotton College, Guwahati 781001, India \\ Correspondence should be addressed to Debojit Sarma; sarma.debojit@gmail.com
}

Received 17 August 2016; Revised 13 October 2016; Accepted 13 October 2016

Academic Editor: Luis A. Anchordoqui

Copyright ( 2016 Debojit Sarma et al. This is an open access article distributed under the Creative Commons Attribution License, which permits unrestricted use, distribution, and reproduction in any medium, provided the original work is properly cited. The publication of this article was funded by SCOAP S $^{3}$

\begin{abstract}
We present an axially symmetric, asymptotically flat empty space solution of the Einstein field equations containing a naked singularity. The space-time is regular everywhere except on the symmetry axis where it possesses a true curvature singularity. The space-time is of type D in the Petrov classification scheme and is locally isometric to the metrics of case IV in the Kinnersley classification of type D vacuum metrics. Additionally, the space-time also shows the evolution of closed timelike curves (CTCs) from an initial hypersurface free from CTCs.
\end{abstract}

\section{Introduction}

The weak cosmic censorship hypothesis (WCCH) of Penrose forbids naked singularities to be the end state of gravitational collapse $[1,2]$. This statement, although accepted by many, has been neither proved nor disproved and, as a matter of fact, recent studies have shown that naked singularities can be formed in a continual gravitational collapse of a dust cloud beginning from a regular initial data [3], indicating therefore the possibility of naked singularities occurring in nature. Since proving (or disproving) the $\mathrm{WCCH}$ has proved to be a difficult task, attempts have been made to provide the theoretical framework to devise a technique to distinguish between black holes and naked singularities from astrophysical data mainly through gravitational lensing. Some significant works in this direction are the study of strong gravitational lensing in the Janis-Newman-Winicour spacetime $[4,5]$ and its rotating generalization [6] and notably (although not an exhaustive list) the work in [7-10]. Other workers have shown that naked singularities and black holes may be differentiated by the properties of the accretion disks that accumulate around them ([11] and references therein). Consequently, the study of naked singularities and spacetime with such objects is of considerable current interest.

In [11], the authors have enumerated three possible end states of gravitational collapse. Of these, the last one, namely, that matter falls into the space-time singularity preserving its symmetry structure and leading to a final configuration that is vacuum space-time with a singularity that is either naked or covered, is of special interest to us. In this paper, we attempt to construct such space-time-a vacuum solution of Einstein's equations with a naked singularity. In what follows, we construct a Ricci flat metric with a singularity at $r=0, r$ being the radial coordinate, and show that the space-time is axially symmetric and asymptotically flat. In addition, we find that the metric admits closed timelike curves which appear at a certain moment, implying that it also represents time machine space-time.

Closed timelike curves (CTCs), closed timelike geodesics (CTGs), and closed null geodesics (CNGs), which all imply violation of causality, are an intriguing aspect of the general theory of relativity and are present in certain solutions of Einstein's field equations. Beginning with Gödel's universe [12], a considerable number of such causality violating solutions have been constructed. van Stockum's solution [13], found years before Gödel's, was later shown to possess CTCs [14]. A few other examples of CTC space-time are Bonnor's rotating dust space-time which has been analysed in [15] and found to have CTCs, Gott's [16] space-time with two moving cosmic strings and wormhole space-time [17], and a pure radiation metric with CTCs [18]. In fact, over the years, a number of solutions with CTCs have been obtained. CTCs have also 
been studied in the context of Brans-Dicke gravity [19], where rigid rotation of matter has been shown to generate these closed curves. Ori and coworkers [20-23] have obtained solutions with CTCs which appear after a certain instant, thereby raising the possibility of constructing a workable time machine at least in theory. We place space-time with CTCs (or CTGs or CNGs) into two categories. In the first category, there is the eternal time machine space-time, such as Gödel's universe where CTCs preexist, while, in the second, there are true time machines where CTCs appear at a certain moment.

Solutions with CTGs and CNGs have also been discussed in the literature. Soares found a class of cosmological solutions with rotating dust and electromagnetic fields admitting [24]. Bonnor and Steadman [25] have shown that space-time with two spinning particles, under special circumstances, allows for the existence of CTGs. A class of solutions of Einstein's field equations with CTGs representing space-time outside a spinning cosmic string surrounded by a region of finite radial extension with vacuum energy and a gas of strings [26] have been recently found. Other examples are cylindrically symmetric space-time [27] which admit CTGs everywhere outside an axis and vacuum space-time with CNGs found quite recently [28].

\section{Space-Time with a Singularity}

Consider the following line element, empty space-time, given by

$$
\begin{aligned}
d s^{2}= & -\sinh ^{2} r \cosh t \operatorname{coth} t d t^{2}+\cosh ^{2} r \sinh r d r^{2} \\
& +\operatorname{csch} r d z^{2} \\
& +\sinh ^{2} r\left(2 \sqrt{2} \cosh t d t d \phi-\sinh t d \phi^{2}\right) .
\end{aligned}
$$

The coordinates are labelled $x^{1}=r, x^{2}=\phi, x^{3}=z$, and $x^{4}=t$ and their ranges are $r>0,-\infty<z<\infty,-\infty<$ $t<\infty$ and $\phi$ is periodic coordinate $\phi \sim \phi+\phi_{0}$, with $\phi_{0}>0$. The metric is Lorentzian with signature $(+,+,+,-)$ and the determinant of the corresponding metric tensor $g_{\mu \nu}$, det $g=$ $-\cosh ^{2} r \sinh ^{4} r \cosh ^{2} t$. The metric (1) is a solution of Einstein's equations in vacuum satisfying the equation $R_{\mu \nu}=0$.

That the space-time represented by (1) is axisymmetric is clear from the following. Consider Killing vector $\eta=\partial_{\phi}$ having the normal form:

$$
\eta^{\mu}=(0,1,0,0) .
$$

The corresponding covector is

$$
\eta_{\mu}=\sinh ^{2} r(0,-\sinh t, 0, \sqrt{2} \cosh t) .
$$

The vector (2) satisfies Killing equation $\eta_{\mu ; \nu}+\eta_{v ; \mu}=0$. Space-time is cyclically symmetric if it admits a Killing vector with spacelike, closed orbits. Axial symmetry means that the space-time contains a nonempty axis of symmetry. The presence of this axis of symmetry is ensured if the norm of $\eta^{\mu}$ vanishes on the axis, that is, at $r=0$ (see $[29,30]$ and references therein). In our case, we find that the norm is

$$
\eta_{\mu} \eta^{\mu}=-\sinh t \sinh ^{2} r
$$

which represents spacelike, closed orbits for $t<0$ and vanishes on the symmetry axis at $r \rightarrow 0$. The norm of $\eta_{\mu}$ changes sign for $t>0$ implying the formation of closed timelike curves which are the subject of the next section.

The metric has a curvature singularity at $r=0$. We find that the Kretschmann scalar (1)

$$
K=R^{\mu \nu \rho \sigma} R_{\mu \nu \rho \sigma}=12 \operatorname{csch}^{6} r
$$

and its differential invariant

$$
R_{\mu \nu \rho \sigma ; \lambda} R^{\mu \nu \rho \sigma ; \lambda}=180 \operatorname{csch}^{9} r
$$

blow up at $r=0$, indicating a curvature singularity. We also note that both these scalar invariants approach zero rapidly as $r$ increases.

The metric (1) belongs to type D in the Petrov classification scheme. To show this, we construct the following set of null tetrads $k, l, m, \bar{m}$ for the metric (1). Explicitly, these vectors have the following form:

$$
\begin{aligned}
k^{\mu} & =\operatorname{csch} r\left(0, \frac{1}{\sqrt{2}} \operatorname{csch} t, 0, \frac{1}{2}(2+\sqrt{2}) \operatorname{sech} t\right), \\
l^{\mu} & =\operatorname{csch} r\left(0,-\frac{1}{\sqrt{2}}, 0, \frac{1}{2}(-2+\sqrt{2}) \tanh t\right), \\
m^{\mu} & =\frac{1}{\sqrt{2 \sinh r}}(\operatorname{sech} r, 0, i \sinh r, 0), \\
\bar{m}^{\mu} & =\frac{1}{\sqrt{2 \sinh r}}(\operatorname{sech} r, 0,-i \sinh r, 0),
\end{aligned}
$$

where $i=\sqrt{-1}$. The set of null tetrads above is such that the metric tensor for the line element (1) can be expressed as

$$
g_{\mu \nu}=-k_{\mu} l_{v}-l_{\mu} k_{\nu}+m_{\mu} \bar{m}_{\nu}+\bar{m}_{\mu} m_{\nu} .
$$

The vectors (7) are null vectors and are orthogonal, except for $k_{\mu} l^{\mu}=-1$ and $m_{\mu} \bar{m}^{\mu}=1$.

Using the set of null tetrads above, we find that, of the five Weyl scalars, only

$$
\Psi_{2}=C_{\mu \nu \rho \sigma} k^{\mu} m^{\nu} \bar{m}^{\rho} l^{\sigma}=\frac{1}{2} \operatorname{csch}^{3} r
$$

is nonzero. The metric is clearly of type $\mathrm{D}$ in the Petrov classification scheme. It clear from (5), (6), and (9) that these scalars rapidly vanish as $r \rightarrow \infty$ leading to the conclusion that the metric is asymptotically flat. In fact, all nonzero curvature invariants for the space-time have form $A \operatorname{csch}^{n} r, A$ being a real constant and $n$ a positive integer which means that their asymptotic behaviour is the same as that of the scalars in (5), (6), and (9).

Vector $k^{\mu}$ is not only null but also geodesic, satisfying $k_{\mu ; \nu} k^{\nu}=0$. We have calculated the kinematical properties of 
the metric such as expansion, shear, and twist and they are given by

$$
\begin{aligned}
\boldsymbol{\Theta} & =\frac{1}{2} k_{; \mu}^{\mu}=0, \\
\boldsymbol{\omega}^{2} & =\frac{1}{2}\left(k_{\mu ; \nu}-k_{\nu ; \mu}\right) k^{\mu ; \nu}=0, \\
\boldsymbol{\sigma} \overline{\boldsymbol{\sigma}} & =\frac{1}{2}\left(k_{\mu ; \nu}+k_{\nu ; \mu}\right) k^{\mu ; \nu}-\Theta^{2}=0 .
\end{aligned}
$$

Hence, the space-time admits expansion-free, twist-free, and shear-free null geodesic congruence.

Type D vacuum space-time is known and has been classified by Kinnersley [31]. A calculation of the NewmanPenrose spin coefficients [32] shows that, for our case, the spin coefficient $\rho=0$. Accordingly, the space-time will be placed in case IV of the classification scheme in [31].

It is interesting property of the metric (1) that it reduces to the Misner space [33] in two space-time dimensions. To demonstrate this, we first apply transformations $t \rightarrow \sinh ^{-1} t$ followed by $\phi \rightarrow \phi+\sqrt{2} \ln t$, to get

$$
\begin{aligned}
d s^{2}= & \cosh ^{2} r \sinh r d r^{2}+\operatorname{csch} r d z^{2} \\
& +\sinh ^{2} r\left(-t d \phi^{2}+\frac{1}{t} d t^{2}\right) .
\end{aligned}
$$

A further transformation into (11) by

$$
\phi \longrightarrow \phi+\ln t
$$

yields

$$
\begin{aligned}
d s^{2}= & \cosh ^{2} r \sinh r d r^{2}+\operatorname{csch} r d z^{2} \\
& -\sinh ^{2} r\left(2 d t d \phi+t d \phi^{2}\right) .
\end{aligned}
$$

The above reduces to the Misner space for constants $r$ and $z$, indicating that the metric discussed here is $4 \mathrm{D}$ extension of the Misner space.

\section{CTCs and Cosmic Time Machines}

A peculiar property of the space-time (1) is that it generates CTCs at a certain moment exhibiting time machine-like behaviour. Consider closed orbits of constant $r=r_{0}>0$, $z=z_{0}$, and $t=t_{0}$ where $r_{0}, z_{0}$, and $t_{0}$ are constants given by 1D line element:

$$
d s^{2}=g_{\phi \phi} d \phi^{2}=-\sinh t \sinh ^{2} r d \phi^{2} .
$$

These orbits are null curves at $t=t_{0}=0$, spacelike throughout $t=t_{0}<0$, but become timelike; that is, $g_{\phi \phi}<0$ for $t=t_{0}>0$, which indicates the presence of CTCs. Hence, CTCs form at an instant of time satisfying $t=t_{0}>0$.

That these CTCs evolve from initially spacelike $t=$ constant hypersurface can be determined by calculating the norm of vector $\nabla_{\mu} t$ (or alternately from the value of $g^{t t}$ in inverse metric tensor $\left.g^{\mu \nu}\right)$. A hypersurface $t=$ constant is spacelike when $g^{t t}<0$, timelike when $g^{t t}>0$, and null when $g^{t t}=0$. From the metric given in (1),

$$
g^{t t}=\sinh t \operatorname{sech}^{2} t \operatorname{csch}^{2} r .
$$

Thus, a hypersurface $t=$ constant is spacelike for $t<0$, timelike for $t>0$, and null at $t=0$. Thus, the spacelike $t=$ constant $<0$ hypersurface can be chosen as initial hypersurface over which initial data may be specified. There is a Cauchy horizon at $t=0$, called chronology horizon, which separates the causal past and future in a past directed and a future directed manner. Hence, the space-time evolves from a partial Cauchy surface (i.e., Cauchy spacelike hypersurface) into a null hypersurface that is causally well-behaved up to a moment, that is, a null hypersurface $t=0$, and the formation of CTCs takes place from causally well-behaved initial conditions. This type of CTCs is different from those in the Gödel universe [34], where the CTCs preexist, and similar to those in [21], where they form at some moment. The CTCs of the space-time discussed in this paper are analogous to those formed in the Misner space [33]. The metric for the Misner space in 2D is

$$
d s_{\text {Mis }}^{2}=-2 d T d X-T d X^{2},
$$

where $-\infty<T<\infty$, but the coordinate $X$ is periodic. The curves $T=T_{0}$, where $T_{0}$ is a constant, are closed since $X$ is periodic. The curves $T<0$ are spacelike and $T>0$ are timelike, while the null curves $T=0$ form the chronology horizon. The second type of curves, namely, $T>0$, is closed timelike curves (CTCs).

An interesting possible interpretation of the metric (1) is in terms of a cosmic time machine discussed by Clarke and de Felice. They have shown [35] that if continued gravitational collapse leads to a strong curvature singularity, then strong causality is violated. This would mean that the entire space-time becomes causally ill-behaved and a cosmic time machine is the result. They define a cosmic time machine as space-time which is asymptotically flat and admits closed nonspacelike curves [36]. Our space-time may represent such a cosmic time machine.

\section{Competing Interests}

The authors declare that there are no competing interests regarding publication of this paper.

\section{References}

[1] R. Penrose, "The question of cosmic censorship," in Black Holes and Relativistic Stars, R. M. Wald, Ed., chapter 5, The Chicago University Press, Chicago, Ill, USA, 1998.

[2] R. M. Wald, "Gravitational collapse and cosmic censorship," https://arxiv.org/abs/gr-qc/9710068.

[3] P. S. Joshi and D. Malafarina, "Recent developments in gravitational collapse and spacetime singularities," International Journal of Modern Physics D, vol. 20, no. 14, p. 2641, 2011.

[4] K. S. Virbhadra, D. Narasimha, and S. M. Chitre, "Role of the scalar field in gravitational lensing," Astronomy and Astrophysics, vol. 337, no. 1, pp. 1-8, 1998. 
[5] K. S. Virbhadra and G. F. Ellis, "Gravitational lensing by naked singularities," Physical Review D, vol. 65, no. 10, Article ID 103004, 2002.

[6] G. N. Gyulchev and S. S. Yazadjiev, "Gravitational lensing by rotating naked singularities," Physical Review D, vol. 78, no. 8, Article ID 083004, 2008.

[7] M. C. Werner and A. O. Petters, "Magnification relations for Kerr lensing and testing cosmic censorship," Physical Review D, vol. 76, no. 6, Article ID 064024, 6 pages, 2007.

[8] C. Bambi and N. Yoshida, "Shape and position of the shadow in the $\delta=2$ Tomimatsu-Sato spacetime," Classical and Quantum Gravity, vol. 27, no. 20, Article ID 205006, 2010.

[9] C. Bambi and K. Freese, "Apparent shape of super-spinning black holes," Physical Review D. Particles, Fields, Gravitation, and Cosmology, vol. 79, no. 4, Article ID 043002, 2009.

[10] K. Hioki and K.-I. Maeda, "Measurement of the Kerr spin parameter by observation of a compact object's shadow," Physical Review D, vol. 80, Article ID 024042, 2009.

[11] A. N. Chowdhury, M. Patil, D. Malafarina, and P. Joshi, "Circular geodesics and accretion disks in the Janis-NewmanWinicour and gamma metric spacetimes," Physical Review D, vol. 85, no. 10, Article ID 104031, 2012.

[12] K. Gödel, "An example of a new type of cosmological solutions of Einstein's field equations of gravitation," Reviews of Modern Physics, vol. 21, pp. 447-450, 1949.

[13] W. J. van Stockum, “The gravitational field of a distribution of particles rotating about an axis of symmetry," Proceedings of the Royal Society of Edinburgh, vol. 57, pp. 135-154, 1937.

[14] F. J. Tipler, "Rotating cylinders and the possibility of global causality violation," Physical Review D, vol. 9, pp. 2203-2206, 1974.

[15] P. Collas and D. Klein, "Letter: causality violating geodesics in bonnor's rotating dust metric," General Relativity and Gravitation, vol. 36, no. 11, pp. 2549-2557, 2004.

[16] I. R. Gott, "Closed timelike curves produced by pairs of moving cosmic strings: exact solutions," Physical Review Letters, vol. 66, no. 9, pp. 1126-1129, 1991.

[17] M. S. Morris, K. S. Thorne, and U. Yurtsever, "Wormholes, time machines, and the weak energy condition," Physical Review Letters, vol. 61, no. 13, pp. 1446-1449, 1988.

[18] D. Sarma, M. Patgiri, and F. U. Ahmed, "Pure radiation metric with stable closed timelike curves," General Relativity and Gravitation, vol. 46, article 1633, 2014.

[19] L. A. Anchordoqui, S. E. P. Bergliaffa, M. L. Trobo, and G. S. Birman, "Cylindrically symmetric spinning Brans-Dicke spacetimes with closed timelike curves," Modern Physics Letters A, vol. 14, no. 17, pp. 1105-1111, 1999.

[20] A. Ori, "Formation of closed timelike curves in a composite vacuum/dust asymptotically flat spacetime," Physical Review D. Particles, Fields, Gravitation, and Cosmology, vol. 76, no. 4, Article ID 044002, 2007.

[21] A. Ori, "A class of time-machine solutions with a compact vacuum core," Physical Review Letters, vol. 95, no. 2, Article ID 021101, 2005.

[22] Y. Soen and A. Ori, "Improved time-machine model," Physical Review D, vol. 54, no. 8, pp. 4858-4861, 1996.

[23] A. Ori and Y. Soen, "Causality violation and the weak energy condition," Physical Review D, vol. 49, no. 8, pp. 3990-3997, 1994.

[24] I. D. Soares, "Inhomogeneous rotating universes with closed timelike geodesics of matter," Journal of Mathematical Physics, vol. 21, no. 3, pp. 521-525, 1980.
[25] W. B. Bonnor and B. R. Steadman, "Exact solutions of the Einstein-Maxwell equations with closed timelike curves," General Relativity and Gravitation, vol. 37, no. 11, pp. 1833-1844, 2005.

[26] Ø. Grøn and S. Johannesen, "Closed timelike geodesics in a gas of cosmic strings," New Journal of Physics, vol. 10, Article ID 103025, 2008.

[27] Ø. Grøn and S. Johannesen, "A spacetime with closed timelike geodesics everywhere," Nuovo Cimento della Societa Italiana di Fisica B, vol. 125, no. 10, pp. 1215-1221, 2010.

[28] D. Sarma, M. Patgiri, and F. U. Ahmed, "A vacuum spacetime with closed null geodesics," Annals of Physics, vol. 329, pp. 179184, 2013.

[29] M. Mars and J. M. M. Senovilla, "Axial symmetry and conformal Killing vectors," Classical and Quantum Gravity, vol. 10, no. 8, pp. 1633-1647, 1993.

[30] M. Mars and J. M. M. Senovilla, "Comment on 'an infinite perfect fluid in cylindrically symmetric steady differential rotation"' Classical and Quantum Gravity, vol. 12, p. 2071, 1995.

[31] W. Kinnersley, “Type D vacuum metrics," Journal of Mathematical Physics, vol. 10, pp. 1195-1203, 1969.

[32] H. Stephani, D. Kramer, M. MacCallum, C. Hoenselaers, and E. Herlt, Exact Solutions to Einstein's Field Equations, Cambridge University Press, Cambridge, UK, 2003.

[33] C. W. Misner, "Taub-nut space as a counterexample to almost anything," in Relativity Theory and Astrophysics I: Relativity and Cosmology, J. Ehlers, Ed., vol. 8 of Lectures in Applied Mathematics, American Mathematical Society, 1967.

[34] K. Gödel, "An example of a new type of cosmological solutions of Einstein's field equations of gravitation," Reviews of Modern Physics, vol. 21, no. 3, pp. 447-450, 1949.

[35] C. J. Clarke and F. de Felice, "Globally noncausal space-times. II. Naked singularities and curvature conditions," General Relativity and Gravitation, vol. 16, no. 2, pp. 139-148, 1984.

[36] F. de Felice, "Cosmic time machines: the causality issue," EPJ Web of Conferences, vol. 58, Article ID 01001, 4 pages, 2013. 

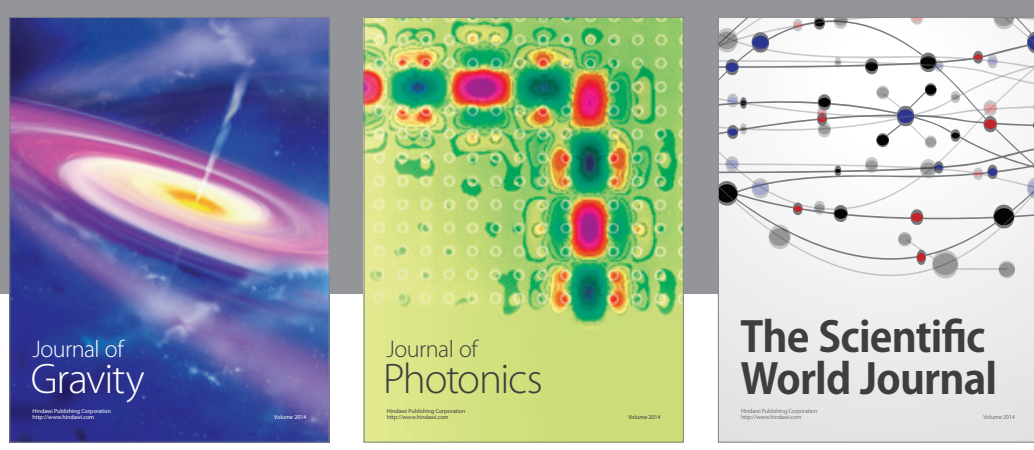

The Scientific World Journal
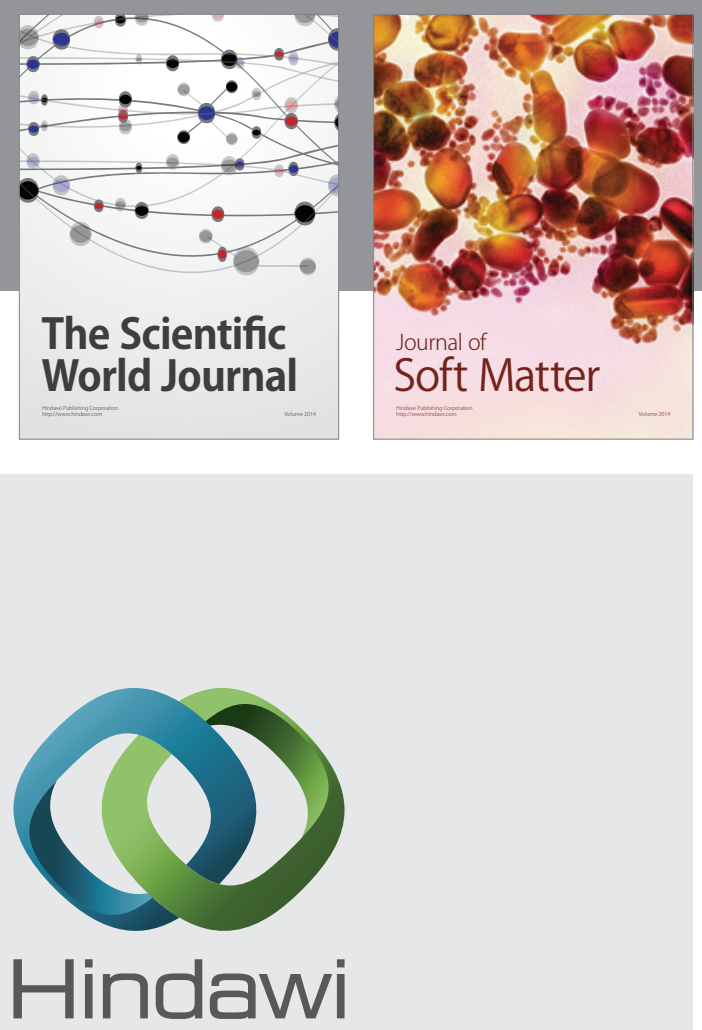

Submit your manuscripts at

http://www.hindawi.com

nternational Journal of

Statistical Mechanics
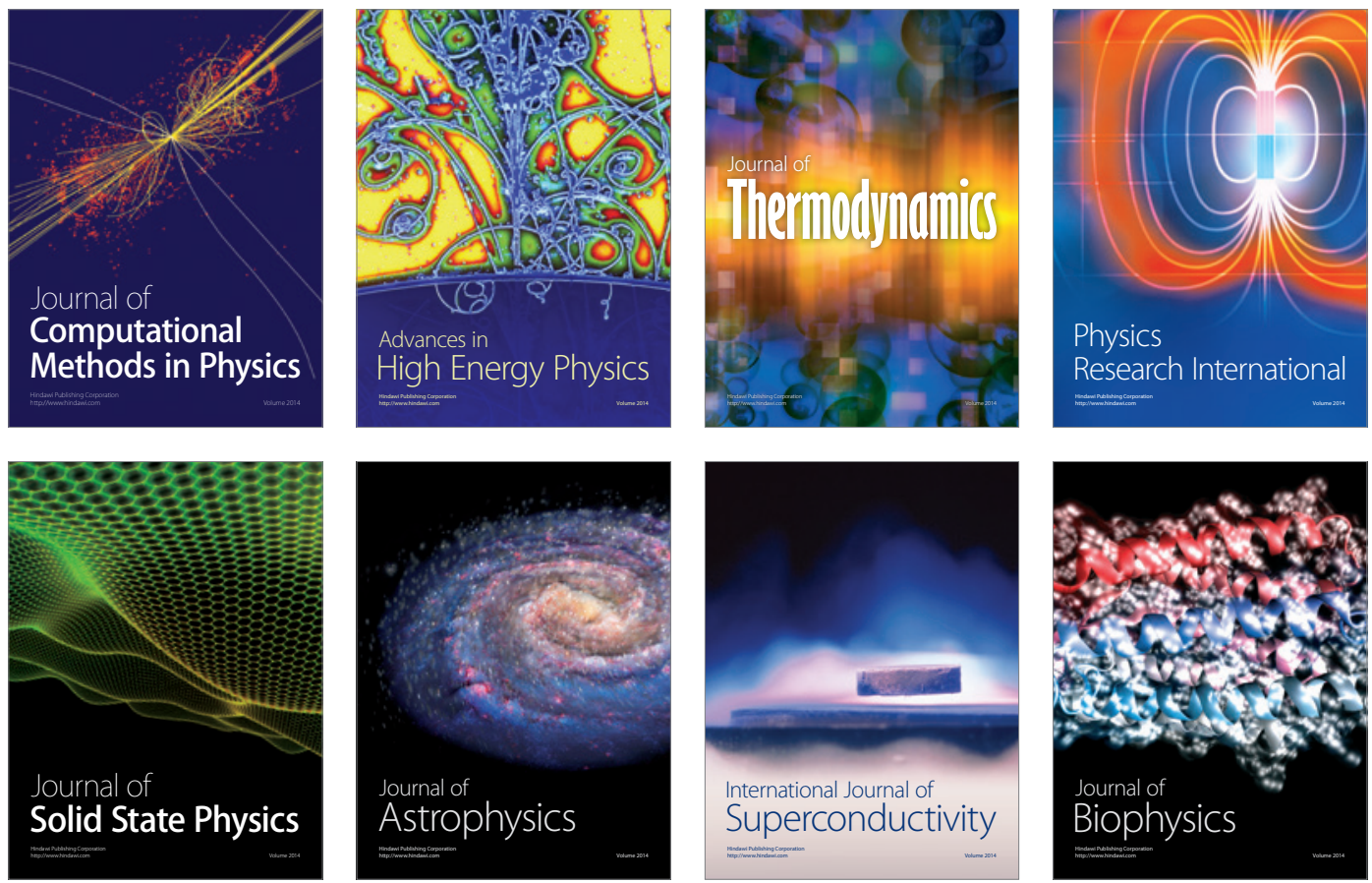
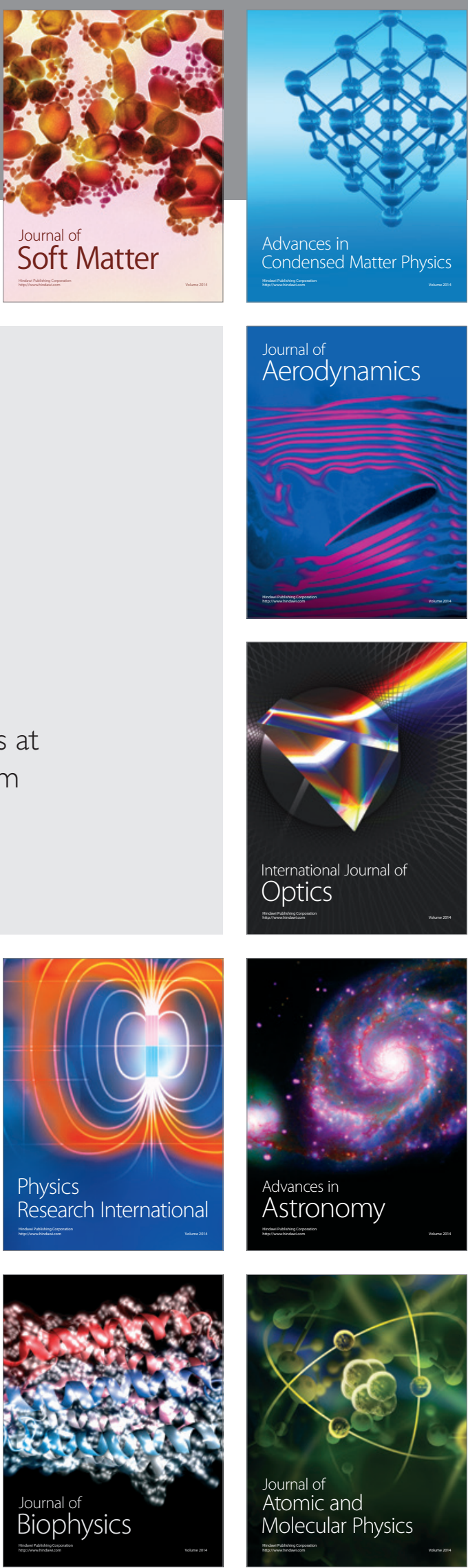